\title{
OBSTETRIC OUTCOMES IN TAKAYASU ARTERITIS
}

\author{
Ana Luisa Souza Pedreira ${ }^{1,{ }^{*}}$, Giovana Pontes Chagas $^{1}$ \\ 1. Escola Bahiana de Medicina e Saúde Pública, Salvador (BA), Brasil. \\ *Corresponding author: anapedreira@bahiana.edu.br
}

\section{BACKGROUND}

Takayasu arteritis (TA) is a large vessel vasculitis that affects women of childbearing age. Although cardiovascular and hemodynamic changes in pregnancy represent a potential risk, studies are still conflicting regarding higher risk in maternal and fetal outcomes. The objective of this study was to determine the prevalence of pregnancies and their outcomes in patients with TA.

\section{MATERIALS AND METHODS}

A cross-sectional study was carried out at a public service in Salvador (BA), Brazil. Female patients aged more than 18 years, diagnosed with TA based on the 1990 American College of Rheumatology criteria were included. Epidemiological data and information about obstetric outcomes were collected. Data analysis was made using the Statistical Package for the Social Sciences v.21.0 and the results are expressed as median and interquartile ranges.

\section{RESULTS}

Twenty patients were included. The median age was 38 years (28-48) and the median age of TA diagnosis was 26 years (21-37). Thirteen patients had at least one pregnancy reported (prevalence of 65\%) and the average of pregnancies per patient was 2.92 . Takayasu arteritis was diagnosed after pregnancy in 12 patients and only one before pregnancy. We found a total of 38 pregnancies, 12 spontaneous abortions, and 26 deliveries (20 vaginal and 6 cesareans), with a live birth rate of $68.4 \%$. Table 1 shows the obstetric complications in our sample. There was no maternal or intrauterine death.

Table 1. Obstetric complications in patients with Takayasu arteritis in Salvador (BA), Brazil.

\begin{tabular}{|c|c|c|}
\hline Variables & Pregnancies $(n)$ & $\%$ \\
\hline Spontaneous abortions & 12 & 31.5 \\
\hline Eclampsia/Preeclampsia & 2 & 5.26 \\
\hline Preterm birth & 3 & 7.89 \\
\hline Maternal cardiac arrest during delivery & 1 & 2.63 \\
\hline Postpartum hemorrhage & 2 & 5.26 \\
\hline Previous placenta & 1 & 2.63 \\
\hline
\end{tabular}

\section{DISCUSSION}

While most other studies describe live birth rates around $80 \%$, our rate was $68.4 \%$, probably due to a slightly higher rate of spontaneous abortions. Compared to the literature, we found a lower rate of eclampsia and preeclampsia. Faced with the known fact that there is an important delay in TA diagnosis, our study raises the hypothesis that women are subject to conception without knowing the disease and without specific treatment. 


\section{CONCLUSION}

The prevalence of pregnancies in our sample was $65 \%$ and only 1 patient became pregnant after TA diagnosis. Spontaneous abortion was the most common complication. The study of reproductive health in TA can help to identify maternal and fetal risks and what is the best management for these patients. 